\title{
HELIUM ABUNDANCE FROM RADIO RECOMBINATION LINES
}

\author{
(Abstract) \\ E. KRÜGEL \\ Universitäts Sternwarte, Göttingen, F.R.G.
}

\begin{abstract}
When stars are losing mass some of the material from which they were formed is being returned to the interstellar medium but, owing to stellar evolution, with a somewhat different chemical composition. This material may again be used in star formation. The chemistry of this new generation of stars can be determined with the help of radio recombination lines arising in the ionized gas, which surrounds the newborn stars of early type. At radio wavelengths the brighter ones of these regions may be traced anywhere in the galactic plane. Assuming a primeval Heabundance observed deviations from this primeval value may be explained by mass loss from stars during their evolved stages. This work has been carried out by Mezger and collaborators at Bonn.
\end{abstract}

Unfortunately, in order to interpret radio recombination lines in terms of abundances one must know the ratio of the volume occupied by ionized $\mathrm{He}$ to that occupied by ionized $\mathrm{H}$. Model calculations which I carried out show that in the absence of dust the relation between this ratio and the effective temperature of the exciting star is a very simple one and independent of the particular density distribution of the gas around the star. But dust will generally be present and it has the unpleasant effect of complicating this relation and making it sensitive to the density distribution.

Additional information may be gained from the IR-data. Dust is believed to absorb stellar radiation and reemit it in the IR. Now one can construct a model of the ionized region which fits the observed radio and IR-data. The theoretical value of the model for the ratio of the ionized $\mathrm{He}$ to the ionized $\mathrm{H}$-volume and the then readily derived He-abundance should not be too erroneous.

So a better knowledge of the absorbing properties of the dust and more radio and IR-measurements may help to determine the overall He-abundance. 